\title{
A FLORISTIC INVENTORY OF A SAND HILLS AREA NEAR SASKATOON, SASKATCHEWAN
}

BOHDAN PYLYPEC, Department of Crop Science and Plant Ecology, University of Saskatchewan, Saskatoon, Saskatchewan. S7N 0W0

As the last glacial ice retreated across Saskatchewan some 17,000 to 10,000 years ago, a number of glacial lakes formed along the ice front. ${ }^{7}$ Sandy deltas originated where water flowed into these lakes. After the lakes disappeared, wind action modified the deltas into dune fields such as the Dundurn Sand Hills which originated along the southern shore of glacial Lake Saskatchewan that existed in the Saskatoon area 14,000 to 11,000 years ago.

Most of the Dundurn Sand Hills have been stabilized by vegetation though small areas still exist where wind erosion and deposition are altering landforms, particularly under disturbed conditions such as those incurred under heavy grazing in times of drought. The complex mosaic of plant communities in the Dundurn Sand Hills reflects the physiographic pattern resulting from present and past geomorphic processes. Four major physiographic areas are present ${ }^{4}$ : (i) active complexes where erosion and deposition still occur, (ii) stabilized blowouts (Figure 1) that are saucershaped depressions showing evidence of recent erosion but no erosion at present, (iii) stabilized dunes (Figure 2) which show characteristic dune forms but no evidence of recent erosion, and (iv) dune depressions which are located in stabilized dunes and are distinguished from blowouts in that they show no evidence of recent erosion.

This report summarizes the results of a detailed floristic inventory (1986-1988)

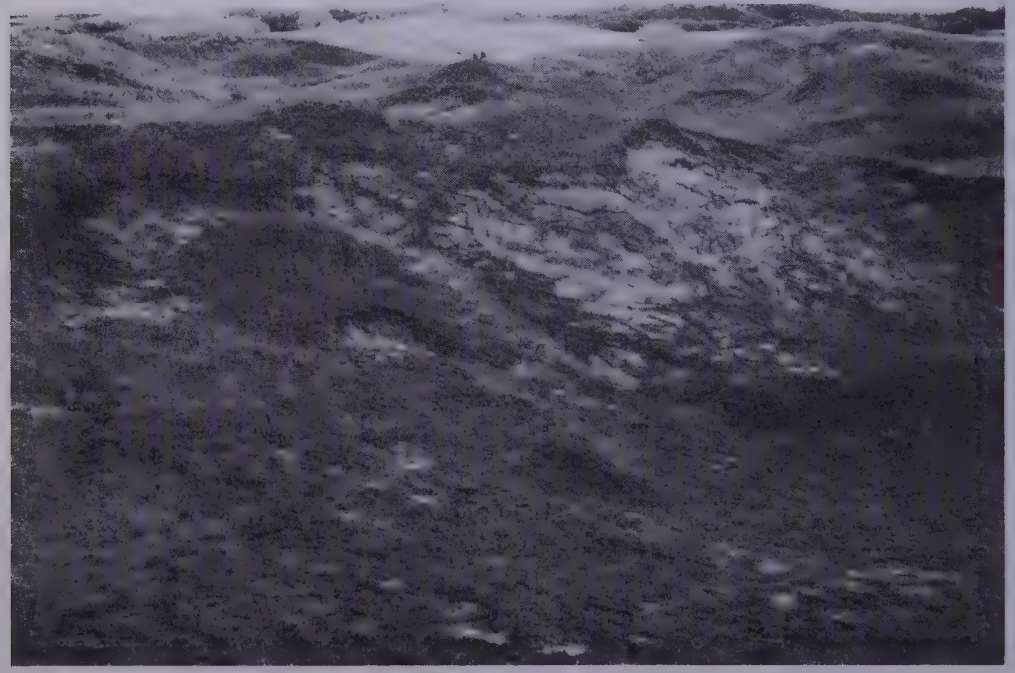

Figure 1. Stabilized blowout covered with mats of Creeping Juniper. B. Pylypec. 


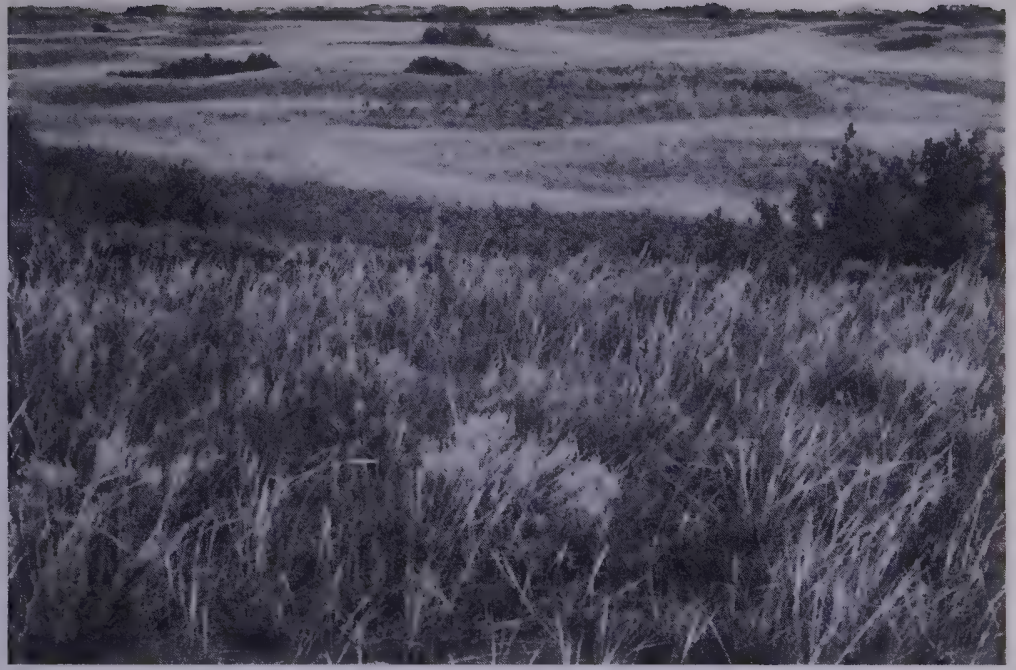

Figure 2. Stabilized dunes covered with Needle-and-thread, Pasture Sage, Plains Wormwood, Western Snowberry and Chokecherry. B. Pylypec.

in the Biddulph Natural Area (E $1 / 212-34-6$ W3), a 121 ha tract of land $25 \mathrm{~km}$ south of Saskatoon owned by the University of Saskatchewan. Habitats present include the four physiographic areas mentioned above and dominated by stabilized dunes, a treed woodland along a spring-fed creek (Figure 3), a marsh meadow in a seepage area at the edge of the dunes (Figure 4), a small beaver pond and several small areas previously cultivated but now partly covered with native vegetation.

Active complexes characterized by drifting sand and sparse vegetation with deep roots and extensive rhizomes are uncommon in the Dundurn Sand Hills, though earlier air photographs (from 1944, for example) reveal these areas were more extensive in the past. Characteristic species of these areas include Prairie Sunflower, Skeletonweed, Lanceleaved Psoralea, Sand Grass, Indian Rice Grass and Northern Wheatgrass. ${ }^{34}$ In the stabilized blowouts many of these pioneer species are replaced by Needleand-thread, June Grass, Sun-loving Sedge, Hairy Golden-aster, Plains Wormwood and Creeping Juniper.
Stabilized dunes constitute the rargest area within the Biddulph Natural Area. Xeric species such as Needle-andthread, Blue Grama, Pasture Sage, Low Sedge, Thread-leaved Sedge, Pricklypear and Purple Prairie-clover are dominant plants. More mesic sites such as slight depressions and north-facing slopes of the dunes are characterized by Chokecherry, Saskatoon, Western Snowberry, Silverberry and Star-flowered Solomon's Seal.

Dune depressions are dominated by Sun-loving Sedge and Western Snowberry. On some sites Plains Rough Fescue and Western Porcupine Grass, usually found in finer-textured soils, are abundant.

The woodland areas are dominated by Trembling Aspen on the upland sites with Balsam Poplar occurring in lower and moister sites. White Birch and River Birch are present along seepage areas often between the two poplar zones. The understory is characterized by species occurring in aspen parklands, e.g., Saskatoon, Chokecherry, Red-osier Dogwood, High Bush-cranberry, Swamp Red Currant, Smooth Wild Strawberry, 


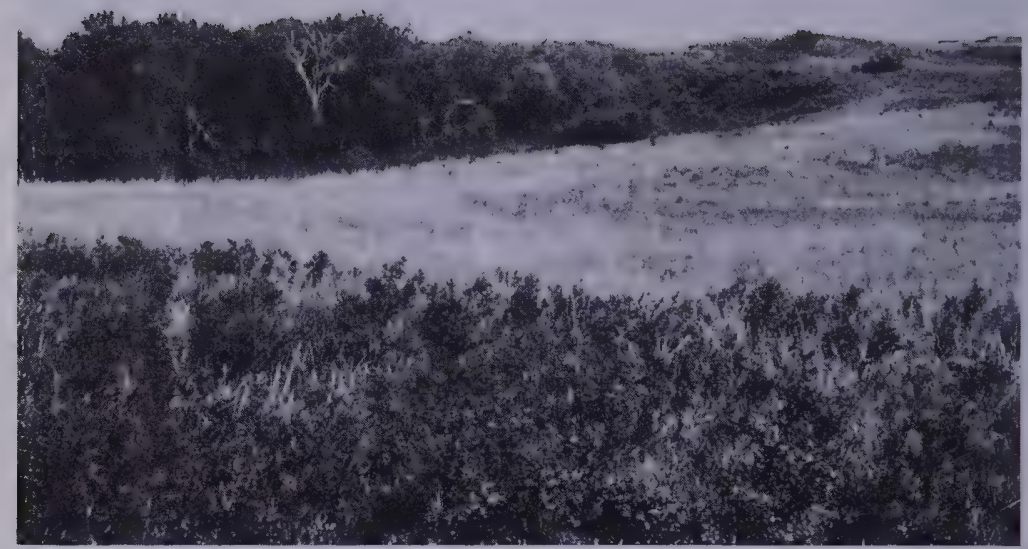

Figure 3. Aspen dominated woodland along spring-fed creek. Needle-and-thread, Western Snowberry and roses in foreground. B. Pylypec.

Cream-coloured Vetchling and Western Canada Violet. Species uncommon elsewhere in similar habitats but relatively abundant here include the Western Red Wood Lily, Small Yellow Lady's-slipper, Green-flowered Bog Orchid and Western Jewelweed.

Species from a wide variety of wetland habitats are present in the Biddulph Natural Area. The small beaver pond has open water species, e.g., Lesser Duckweed, Ivy-leaved Duckweed, surrounded by coarse emergents, e.g., Common Cattail, Common Reed Grass, Tall Manna Grass and Smooth Beggarticks. The creek is lined with species associated with running water and wet meadows, e.g., Brook Grass, Water Sedge, Marsh-marigold, Water-parsnip and Snakeroot. Shallow marsh species are present in the marsh meadow area, e.g., Spangletop, Reed Canary Grass, Silverweed, Mountain Sneezeweed, Beaked Willow and Basket Willow.

Several artificially disturbed areas are examples of secondary plant succession. A borrow pit excavated in 1967 for construction of Highway 219 is now covered with White and Yellow Sweet-clovers (in- troduced), as well as the native pioneer species, Sand Grass. Areas previously cropped by R.C. Biddulph and then seeded to tame forages (Smooth Brome and Crested Wheatgrass) in 1954 and 1955 now also include native species such as Needle-and-thread, Northern Wheatgrass, Western Snowberry, Wood's Rose and Creeping Juniper.

A total of 267 species have been recorded in the Biddulph Natural Area. Voucher specimens have been placed in the W.P. Fraser Herbarium at the University of Saskatchewan.

The wide variety of plant communities represented in this relatively small area, ranging from sparse xeric vegetation on south-facing slopes to mesic woodlands and wetlands, makes this dune area good wildlife habitat. Often areas such as the Biddulph Natural Area are especially valuable since they are surrounded by intensively cultivated agricultural lands. Early man valued these sandy areas, and many of them have numerous interesting archaeological sites. ${ }^{25}$ Other sand dune areas are scattered throughout the province from the Lake Athabasca Dunes in the north with distinctive 


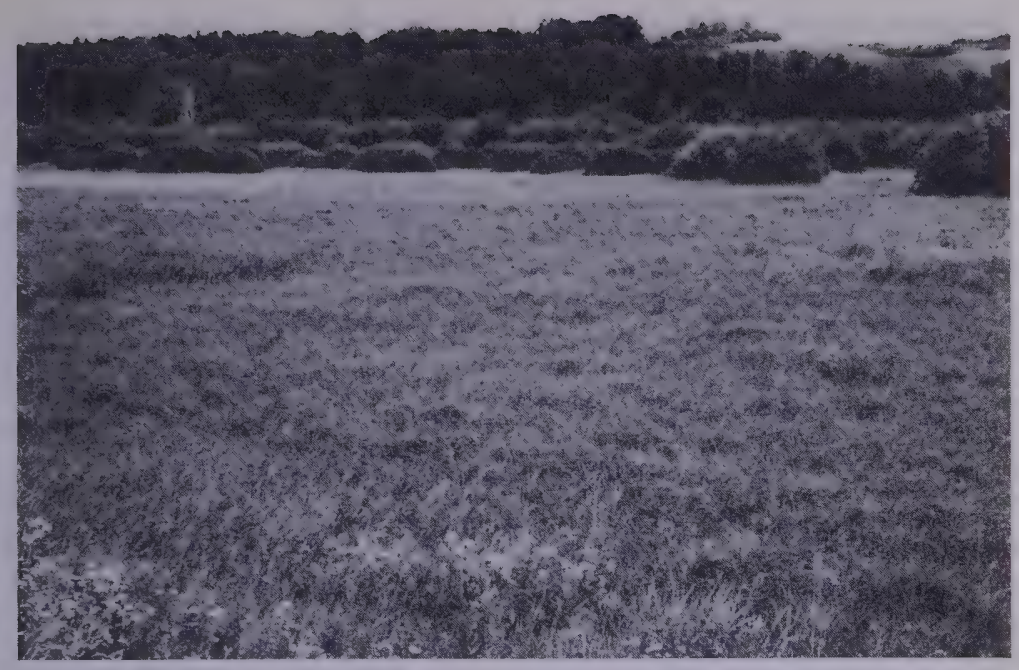

Figure 4. Marsh meadow in seepage area. Sedges and Reed Canary Grass in foreground; willows, Balsam Poplar and Trembling Aspen in background. B. Pylypec.

endemic plants ${ }^{5}$ to the Great Sand Hills of the southwest. The vegetation of the Biddulph Natural Area resembles the Beaver Creek and Cranberry Flats sites located $10-15 \mathrm{~km}$ to the north.

Sand dune areas are exposed to increasingly greater use for consumptive and non-consumptive recreational purposes, cattle grazing and resource extraction, e.g., minerals, oil and gas. Heavy grazing by cattle has a dramatic effect on the plant species composition, leading to decreases in grasses such as Needle-and-thread and increases in Blue Grama, sedges and Prairie Selaginella. Activities such as trampling, all-terrain vehicle use (Figure 5) and grazing can quickly reduce these diverse environments to a simple surface of drifting sand. ${ }^{23}$ The preservation and careful management of these areas often regarded as wastelands by the general public needs to be recognized.

The assistance of V.L. Harms, J.H. Hudson and J.Y. Marchand in the identification of plant specimens is gratefully appreciated. I would also like to thank R.E. Redmann and J.S. Rowe for reviewing the article and B.A. Atimoyoo for typing the manuscript.

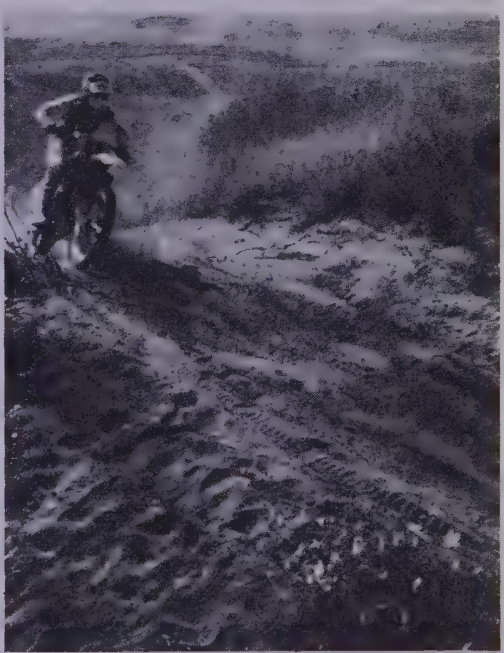

Figure 5. Motocross cycling in Dundurn Sand Hills. J.T. Romo. 
${ }^{1}$ CHRISTIANSEN, E.A. 1979. The Wisconsinan deglaciation of southern Saskatchewan and adjacent areas. Can. J. Earth Sci. 16: 913-938.

${ }^{2}$ EPP, H.T. and L. TOWNLEY-SMITH. 1980. The Great Sand Hills of Saskatchewan. Sask. Dept. of Environment, Regina. 156 pp.

${ }^{3}$ HEATH, J.Y. 1981. The impact of recreational trampling and vehicular traffic on sandhill communities. M.Sc. thesis, University of Saskatchewan, Saskatoon. $174 \mathrm{pp}$.
${ }^{4}$ HULETT, G.K., R.T. COUPLAND and R.L. DIX. 1966. The vegetation of dune sand areas within the grassland region of Saskatchewan. Can. J. Bot. 44: 1307-1331.

${ }^{5}$ ROWE, J.S. and Z.M. ABOUGUENDIA. 1982. The Lake Athabasca Sand Dunes of Saskatchewan: A unique area. The Musk-Ox 30: $1-22$.

\section{CHECKLIST OF THE VASCULAR FLORA OF THE BIDDULPH NATURAL AREA}

PTERIDOPHYTA - Ferns and fern allies

EQUISETACEAE - Horsetail family Equisetum arvense L. - Common horsetail Equisetum hyemale L. var. affine (Engelm.) A.A. Eaton - Common scouring-rush Equisetum laevigatum A.Br. - Smooth scouring-rush

SELAGINELLACEAE - Spike-moss family Selaginella densa Rydb. - Prairie selaginella

SPERMATOPHYTA - Seed-bearing plants

GYMNOSPERMAE - Conifers

CUPRESSACEAE - Cypress family Juniperus communis L. - Low juniper Juniperus horizontalis Moench - Creeping juniper

ANGIOSPERMAE - Flowering plants

MONOCOTYLEDONAE - Monocotyledons

TYPHACEAE - Cattail family

Typha latifolia L. - Common cattail

SPARGANIACEAE - Bur-reed family Sparganium sp. - Bur-reed

ALISMACEAE - Water-plantain family Alisma plantago-aquatica L. - Common water-plantain

POACEAE - Grass family

Agropyron albicans Scribn. \& Smith Awned northern wheatgrass

Agropyron dasystachyum (Hook.) Scribn. var. dasystachyum - Northern wheatgrass
Agropyron dasystachyum (Hook.) Scribn. var. riparium (Scribn. \& Smith) Bowden - Streambank wheatgrass

Agropyron pectiniforme Roem. \& Schult. - Crested wheatgrass

Agropyron repens (L.) Beauv. - Quack grass

Agropyron subsecundum (Link)

Hitchc. - Awned wheatgrass

Agrostis exarata Trin. - Spike redtop

Agrostis scabra Willd. - Rough hair grass

Beckmannia syzigachne (Steud.) Fern. - Slough grass

Bouteloua gracilis (HBK.) Lag. - Blue grama

Bromus ciliatus L. - Fringed brome

Bromus inermis Leyss. - Smooth brome

Calamagrostis canadensis (Michx.)

Beauv. - Marsh reed grass

Calamovilfa longifolia (Hook.) Scribn. - Sand grass

Catabrosa aquatica (L.) Beauv. - Brook grass

Elymus canadensis L. - Canada wild rye

Festuca altaica Trin. ssp. hallii (Vasey)

Harms - Plains rough fescue

Festuca ovina L. - Sheep Fescue

Glyceria grandis S. Wats. - Tall manna grass

Glyceria striata (Lam.) Hitchc. - Fowl manna grass

Helictotrichon hookeri (Scribn.) Henr. - Hooker's oat grass

Hordeum jubatum L. - Wild barley

Koeleria cristata (L.) Pers. - June grass

Muhlenbergia richardsonis (Trin.) Rydb. - Mat muhly

Oryzopsis asperifolia Michx. - Whitegrained mountain rice grass

Oryzopsis hymenoides (Roem. \& Schult.) Ricker - Indian rice grass 
Phalaris arundinacea L. - Reed canary grass

Phragmites communis Trin. - Common reed grass

Poa canbyi (Scribn.) Piper - Canby blue grass

Poa cusickii Vasey - Early blue grass

Poa interior Rydb. - Wood blue grass

Poa pratensis L. - Kentucky blue grass

Poa sandbergii Vasey-Sandberg's blue grass

Schizachne purpurascens (Torr.) SwalIen - Purple oat grass

Schizachyrium scoparium (Michx.) Nash - Little bluestem

Scolochloa festucacea (Willd.) Link Spangletop

Sporobolus cryptandrus (Torr.) A. Gray - Sand dropseed

Stipa comata Trin. \& Rupr. - Needleand-thread

Stipa curtiseta (A.S. Hitchc.) Barkworth - Western porcupine grass

Stipa viridula Trin. - Green needle grass

CYPERACEAE - Sedge family

Carex aquatilis Wahl. - Water sedge

Carex eleocharis Bailey - Low sedge

Carex filifolia Nutt. - Thread-leaved sedge

Carex hystricina Muhl. - Porcupine sedge

Carex obtusata Lilj. - Blunt sedge

Carex pensylvanica Lam. var. digyna Boeckl, - Sun-loving sedge

Carex rostrata Stokes - Beaked sedge

Carex sartwellii Dewey - Sartwell's sedge

Carex siccata Dewey - Hay sedge

Carex sprengelii Dewey - Sprengel's sedge

Carex stipata Muhl. - Awl-fruited sedge

Scirpus microcarpus Pers. - Smallfruited bulrush

Scirpus validus Vahl - Great bulrush

LEMNACEAE - Duckweed family Lemna minor L. - Lesser duckweed Lemna trisulca L. - Ivy-leaved duckweed

JUNCACEAE - Rush family

Juncus balticus Willd. - Baltic rush

LILIACEAE - Lily family

Allium textile Nels. \& Macbr. - Prairie onion

Asparagus officinalis L. - Asparagus

Disporum trachycarpum (S. Wats.) Benth. \& Hook. - Fairybells

Lilium philadelphicum L. var. andinum (Nutt.) Ker. - Western red wood lily

Maianthemum canadense Desf. var. interius Fern. - Two-leaved Solomon's seal
Smilacina stellata (L.) Desf. - Starflowered Solomon's seal

Smilax lasioneuron Hook. - Carrionflower

IRIDACEAE - Iris family

Sisyrinchium montanum Greene Common blue-eyed grass

ORCHIDACEAE - Orchid family

Cypripedium calceolus L. var. parviflorum (Salisb.) Fern. - Small yellow lady's-slipper

Platanthera hyperborea (L.) Lindl. Green-flowered bog orchid

DICOTYLEDONAE - Dicotyledons

SALICACEAE - Willow family

Populus balsamifera L. - Balsam poplar

Populus tremuloides Michx. - Trembling aspen

Salix bebbiana Sarg. - Beaked willow

Salix exigua Nutt. ssp. interior (Rowlee) Cronq. - Sandbar willow

Salix lutea Nutt. - Yellow willow

Salix petiolaris J.E. Smith - Basket willow

BETULACEAE - Birch family

Betula glandulifera (Regel) Butler Swamp birch

Betula occidentalis Hook. - River birch Betula papyrifera Marsh. - White birch

URTICACEAE - Nettle family

Urtica dioca L. var. procera (Muhl.) Wedd. - Stinging nettle

SANTALACEAE - Sandalwood family

Comandra umbellata (L.) Nutt. var. pallida (DC.) M.E. Jones - Pale comandra

POIYGONACEAE - Buckwheat family

Polygonum amphibium L. var. stipulaceum (Coleman) Fern. - Water smartweed

Polygonum aviculare L. - Doorweed

Polygonum convolvulus L. - Wild buckwheat

Rumex occidentalis S. Wats, - Western dock

CHENOPODIACEAE - Goosefoot family

Axyris amaranthoides L. - Russian pigweed

Chenopodium album L. - Lamb's-quarters

Chenopodium pratericola Rydb. - Narrow-leaved goosefoot

Corispermum orientale Lam. var. emarginatum (Rydb.) Macbr. - Villose bugseed 
Salsola kali L. var. tenuifolia Tausch. Russian thistle

NYCTAGINACEAE - Four o'clock family Mirabilis hirsuta (Pursh) MacM. var. hirsuta - Hairy umbrellawort

CARYOPHYLLACEAE - Pink family

Cerastium arvense L. - Field chickweed

Stellaria longifolia Muhl. - Long-leaved stitchwort

Stellaria longipes Goldie - Long-stalked stitchwort

RANUNCULACEAE - Crowfoot family

Actaea rubra (Ait.) Willd. forma neglecta (Gilman) Robins - White baneberry

Actaea rubra (Ait.) Willd. forma rubra Red baneberry

Anemone canadensis L. - Canada anemone

Anemone patens L. var. wolfgangiana (Bess.) Koch - Crocus anemone Anemone riparia Fern. - Tall anemone Caltha palustris L. - Marsh-marigold

Ranunculus glaberrimus Hook. - Shinyleaved buttercup

Ranunculus macounii Britt. - Macoun's buttercup

Ranunculus scleratus L. - Celery-leaved . buttercup

Thalictrum venulosum Trel. - Veiny meadow-rue

BRASSICACEAE - Mustard family

Arabis hirsuta (L.) Scop. var. pycnocarpa (Hopkins) Rollins - Hirsute rock cress

Arabis holboellii Hornem. var. retrofracta (Graham) Rydb. - Reflexed rock cress

Descuraina pinnata (Walt.) Britt. var. brachycarpa (Richards.) Fern. Short-fruited tansy mustard

Descuraina sophia (L.) Webb. - Flixweed

Erysimum asperum (Nutt.) DC. - Western wallflower

Erysimum cheiranthoides L. - Wormseed mustard

Lepidium densiflorum Schrad. - Common pepper-grass

Lesquerella arenosa (Richards.) Rydb. - Sand bladderpod

Rorippa palustris (L.) Besservar. fernaldiana (Butters) Stuckey - Marsh yellow cress

Thlaspi arvense L. - Stinkweed

SAXIFRAGACEAE - Saxifrage family

Heuchera richardsonii R. Br. - Alumroot

Parnassia palustris L. var. tenuis Wahl. - Northern grass-of-parnassus
GROSSULARIACEAE - Currant family

Ribes hudsonianum Richards. - Northern black currant

Ribes oxyacanthoides L. - Northern gooseberry

Ribes triste Pall. - Swamp red currant

ROSACEAE - Rose family

Amelanchier alnifolia Nutt. - Saskatoon

Crataegus chrysocarpa Ashe - Roundleaved hawthorn

Fragaria virginiana Dcne. ssp. glauca (S. Wats.) Staudt - Smooth wild strawberry

Geum aleppicum Jacq. - Yellow avens

Geum triflorum Pursh - Three-flowered avens

Potentilla anserina L. - Silverweed

Potentilla arguta Pursh - White cinquefoil

Potentilla norvegica L. ssp. monspeliensis (L.) Asch. \& Graebn. Rough cinquefoil

Potentilla pensylvanica L. var. atrovirens (Rydb.) T. Wolf - Prairie cinquefoil

Potentilla pensylvanica L. var. pensylvanica - Prairie cinquefoil

Potentilla rivalis Nutt. - Brook cinquefoil

Prunus pensylvanica L.f. - Pincherry

Prunus virginiana L. - Chokecherry

Rosa acicularis Lindl. - Prickly rose

* Rosa arkansana Porter - Low prairie rose

Rosa woodsii Lindl. var. fendleri (Crepin) Rydb. - Wood's rose

Rosa woodsii Lindl. var, terrens (Lunnell) Breitung - Wood's rose

Rosa woodsii Lindl. x Rosa acicularis Lindl. - "Hybrid" rose

Rubus idaeus L. var. strigosus (Michx.) Maxim - Wild red raspberry

Rubus pubescens Raf. - Dewberry

Spiraea alba Du Roi - Narrow-leaved meadowsweet

FABACEAE - Legume family

Astragalus adsurgens Hook. - Ascending purple milk-vetch

Astragalus agrestis Dougl. - Purple milk-vetch

Caragana arborescens Lam. - Common caragana

Glycyrrhiza lepidota (Nutt.) Pursh Wild licorice

Lathyrus ochroleucus Hook. - Creamcoloured vetchling

Lathyrus venosus Muhl. - Wild peavine

Medicago sativa L. - Alfalfa

Melilotus alba Medic. - White sweetclover

Melilotus officinalis (L.) Pall. - Yellow sweet-clover 
Oxytropis campestris (L.) DC. var. gracilis - Late yellow locoweed

Oxytropis sericea Nutt. var. spicata (Hook.) Barneby - Early yellow locoweed

Petalostemon purpureum (Vent.) Rydb. - Purple prairie-clover

Psoralea argophylla Pursh - Silverleaf psoralea

Psoralea esculenta Pursh - Indian breadroot

Psoralea lanceolata Pursh - Lanceleaved psoralea

Thermopsis rhombifolia (Nutt.) Richardson - Golden-bean

Vicia americana Muhl. var. americana - American vetch

Vicia americana Muhl. var. angustifolia Nees - Narrow-leaved vetch

LINACEAE - Flax family

Linum lewisii Pursh - Lewis wild flax

Linum rigidum Pursh - Large-flowered yellow flax

BALSAMINACEAE - Touch-me-not family Impatiens noli-tangere L. - Western jewelweed

ANACARDIACEAE - Sumach family

Rhus radicans L. var. rydbergii (Small) Rehder - Poison-ivy

ACERACEAE - Maple family

Acer negundo L. - Manitoba maple

RHAMNACEAE - Buckthorn family

Rhamnus alnifolia L'Her. - Alder-leaved buckthorn

VIOLACEAE - Violet family

Viola adunca J.E. Smith - Early blue violet

Viola nephrophylla Greene - Northern bog violet

Viola nuttallii Pursh - Nuttall's yellow violet

Viola rugulosa Greene - Western Canada violet

OPUNTIACEAE - Cactus family

Coryphantha vivipara (Nutt.) Britt. \& Rose - Ball cactus

Opuntia fragilis (Nutt.) Haw. - Brittleprickly pear

Opuntia polyacantha Haw. - Pricklypear

ELAEAGNACEAE - Oleaster family

Elaeagnus commutata Bernh. - Silverberry

Shepherdia argentea Nutt. - Buffaloberry
OENOTHERACEAE - Evening-primrose family

Epilobium ciliatum Raf. - Northern willowherb

Gaura coccinea Pursh - Scarlet gaura

Oenothera nuttallii Sweet - White evening-primrose

Oenothera serrulata Nutt. - Shrubby evening-primrose

ARALIACEAE - Ginseng family

Aralia nudicaulis L. - Wild sarsaparilla

APIACEAE - Parsley family

Cicuta maculata L. var. angustifolia Hook. - Water-hemlock

* Cymopterus acaulis (Pursh) Raf. - Plains cymopterus

Osmorhiza longistylis (Torr.) DC. Smooth sweet cicely

Sanicula marilandica L. - Snakeroot

Sium suave Walt. - Water-parsnip

Zizia aptera (Gray) Fern. - Heart-leaved alexanders

CORNACEAE - Dogwood family

Cornus canadensis L. - Bunchberry

Cornus sericea L. ssp. stolonifera (Michx.) Fosberg - Red osier dogwood

PYROLACEAE - Wintergreen family

Pyrola asarifolia Michx. - Pink wintergreen

ERICACEAE - Heath family

Arctostaphylos uva-ursi (L.) Spreng. Bearberry

PRIMULACEAE - Primrose family

Androsace septentrionalis L. - Pygmyflower

Lysimachia ciliata L. - Fringed loosestrife

Lysimachia thyrsiflora L. - Tufted loosestrife

OLEACEAE - Olive family

Fraxinus pennsylvanica Marsh. var. subintegerrima (Vahl.) Fern. - Green ash

APOCYNACEAE - Dogbane family Apocynum androsaemifolium L. var. incanum DC. - Spreading dogbane

ASCLEPIADACEAE - Milkweed family

Asclepias ovalifolia Dcne. - Dwarf milkweed

Asclepias viridiflora Raf. var. linearis (A. Gray) Fern. - Green milkweed

Asclepias viridiflora Raf. var. oborata (EII.) Torrey - Green milkweed 
POLEMONIACEAE - Phlox family

Phlox hoodii Richardson - Moss phlox

BORAGINACEAE - Borage family

Hackelia deflexa (Wahlenb.) Opiz. var. americana (Gray) Fern. \& I.M. Johnston - Nodding stickseed

Lappula redowskii (Hornem.) Greene - Western blue bur

Lithospermum incisum Lehm. - Narrow-leaved puccoon

LAMIACEAE - Mint family

Mentha arvensis L. - Field mint

Monarda fistulosa L. - Wild bergamot

Scutellaria galericulata L. - Marsh skullcap

Stachys palustris L. var. pilosa (Nutt.) Fern. - Marsh hedge-nettle

SCROPHULARIACEAE - Figwort family

Penstemon gracilis Nutt. - Lilacflowered beardtongue

Penstemon nitidus Dougl. - Smooth blue beardtongue

Veronica americana (Raf.) Schwein. American speedwell

* Veronica catenata Pennell - Water speedwell

Veronica scutellata L. - Marsh speedwell

OROBANCHACEAE - Broom-rape family

Orobanche ludoviciana Nutt. Louisiana broom-rape

PLANTAGINACEAE - Plantain family Plantago major L. - Common plantain

RUBIACEAE - Madder family

Galium boreale L. - Northern bedstraw

* Galium trifidum L. - Small bedstraw

Galium triflorum Michx. - Sweetscented bedstraw

CAPRIFOLIACEAE - Honeysuckle family

Linnaea borealis L. \$sp. americana (Forbes) Hulten - Twinflower

Lonicera dioica L. var. glaucescens (Rydb.) Butters. - Twining honeysuckle

Symphoricarpos albus (L.) Blake White snowberry

Symphoricarpos occidentalis Hook. Western snowberry

Viburnum edule (Michx.) Raf. - Low bush-cranberry

Viburnum opulus L. ssp. trilobum Marsh. - High bush-cranberry

CAMPANULACEAE - Bluebell family

Campanula rotundifolia L. - Harebell
ASTERACEAE - Composite family

Achillea lanulosa Nutt. - Common yarrow

Agoseris glauca (Pursh) Raf. - False dandelion

Antennaria aprica Greene - Low everlasting

Artemisia biennis Willd. - Biennial wormwood

Artemisia campestris L. - Plains wormwood

Artemisia frigida Willd. - Pasture sage

Artemisia ludoviciana Nutt. var. gnaphalodes (Nutt.) T.\&G. - Slender sage

Aster ericoides L, ssp. pansus (Blake) A.G. Jones - Many-flowered aster Asterhesperius A. Gray - Willow aster Aster laevis L. ssp. geyeri A. Gray Smooth aster

Bidens cernua L. - Smooth beggarticks

Carduus nutans L. - Nodding thistle

Cirsium arvense (L.) Scop. - Canada thistle

Cirsium flodmanii (Rydb.) Arthur Flodman's thistle

Crepis tectorum L. - Narrow-leaved hawk's-beard

Erigeron asper Nutt. - Rough fleabane

Erigeron caespitosus Nutt. - Tufted fleabane

Erigeron philadelphicus L. - Philadelphia fleabane

Gaillardia aristata Pursh - Greatflowered gaillardia

Grindelia squarrosa (Pursh) Dunal Gumweed

Gutierrezia sarothrae (Pursh) Britt. \& Rusby - Common broomweed

Helenium autumnale L. - Mountain sneezeweed

Helianthus laetiflorus Pers. var. subrhomboideus (Rydb.) Fern. - Beautiful sunflower

Helianthus petiolaris Nutt. - Prairie sunflower

Heterotheca villosa (Pursh) Shinners Hairy golden-aster

Hieracium umbellatum L. - Canada hawkweed

Lactuca pulchella (Pursh) DC. - Blue lettuce

Liatris punctata Hook. - Dotted blazingstar

Lygodesmia juncea (Pursh) D. Don Skeletonweed

Petasites frigidus (L.) Fries var. nivalis (Greene) Cronq. - Vine-leaved colt'sfoot

Petasites sagittatus (Pursh) A. Gray Arrow-leaved colt's foot

Prenanthes racemosa Michx. - Glaucous white lettuce 
Senecio canus Hook. - Silvery groundsel

Senecio integerrimus Nutt. var. integerrimus - Entire-leaved groundsel

Solidago canadensis L. var. gilvocanescens Rydb. - Canescent goldenrod

Solidago gigantea Ait. var. serotina (Ait.) Cronq. - Late goldenrod

Solidago missouriensis Nutt. - Low goldenrod

Solidago nemoralis Ait. var. Iongipetiolata (Mack. \& Bush) - Showy goldenrod

Solidago spathulata DC. var. neomexicana (Rydb.) Cronq. - Mountain goldenrod
Sonchus arvensis L. - Perennial sowhistle

Taraxacum officinale Weber - Common dandelion

Townsendia exscapa (Rich.) Porter Low townsendia

Tragopogon dubius Scop. - Yellow goat's-beard

*Voucher specimens in W.P. Fraser Herbarium collected by earlier investigators.

\section{A SELECTED, ANNOTATED BIBLIOGRAPHY FOR SASKATCHEWAN BUTTERFLY WATCHERS}

\section{BERNIE GOLLOP, 2202 York Avenue, Saskatoon, Saskatchewan. S7J 1J1}

The first part of this article (10 titles) is intended to help Saskatchewan butterfly watchers decide which identification guides will help them most to determine what butterflies they are seeing or catching. Most of these books also treat the ecology of each species, in varying detail. As with bird guides, the more butterfly books one has, the better the chance of identifying the more difficult species. The second part (five titles) deals with butterfly watching and biology in general. Prices are approximate and sometimes vary from store to store; all are available from the Blue Jay Bookshop.

Monarch

Gary Seib

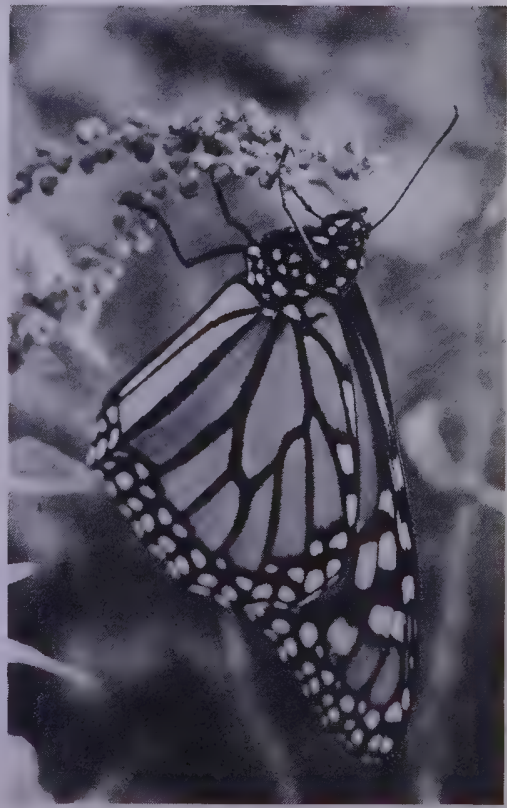

